OPEN ACCESS

Edited by:

Guihua Jiang,

Guangdong Second Provincial

General Hospital, China

Reviewed by:

Patrick K. A. Neff,

University of Regensburg, Germany

Jae-Jin Song,

Seoul National University Bundang

Hospital, South Korea

Xiao-Min Xu,

Nanjing Medical University, China Yuexin Cai,

Sun Yat-sen University, China Yu-Chen Chen,

Nanjing Medical University, China

*Correspondence:

Han LV

chris/vhan@126.com

Zhenchang Wang

cjr.wzhch@vip.163.com

Specialty section:

This article was submitted to Auditory Cognitive Neuroscience, a section of the journal

Frontiers in Neuroscience

Received: 17 May 2021 Accepted: 28 October 2021

Published: 17 November 2021

Citation:

Wei X, Lv H, Chen Q, Wang Z, Zhao P, Liu C, Gong S, Yang Z and

Wang $Z$ (2021) Surface-Based

Amplitude of Low-Frequency

Fluctuation Alterations in Patients With Tinnitus Before and After Sound Therapy: A Resting-State Functional Magnetic Resonance Imaging Study.

Front. Neurosci. 15:709482.

doi: 10.3389/fnins.2021.709482

\section{Surface-Based Amplitude of Low-Frequency Fluctuation} Alterations in Patients With Tinnitus Before and After Sound Therapy: A Resting-State Functional Magnetic Resonance Imaging Study

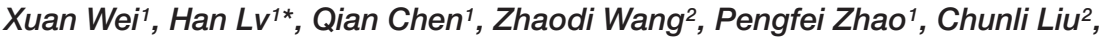 \\ Shusheng Gong ${ }^{2}$, Zhenghan Yang ${ }^{1}$ and Zhenchang Wang ${ }^{1 *}$ \\ ${ }^{1}$ Department of Radiology, Beijing Friendship Hospital, Capital Medical University, Beijing, China, ${ }^{2}$ Department \\ of Otolaryngology Head and Neck Surgery, Beijing Friendship Hospital, Capital Medical University, Beijing, China
}

This study aimed to investigate abnormal tinnitus activity by evaluating brain surfacebased amplitude of low-frequency fluctuation (ALFF) changes detected by resting-state functional magnetic resonance imaging (RS-fMRI) in patients with idiopathic tinnitus before and after 24 weeks of sound therapy. We hypothesized that sound therapy could gradually return cortical local brain function to a relatively normal range. In this prospective observational study, we recruited thirty-three tinnitus patients who had undergone 24 weeks of sound therapy and 26 matched healthy controls (HCs). For the two groups of subjects, we analyzed the spontaneous neural activity of tinnitus patients by cortical ALFF and detected its correlation with clinical indicators of tinnitus. Patients' Tinnitus Handicap Inventory (THI) scores were assessed to determine the severity of their tinnitus before and after treatment. Two-way mixed model analysis of variance and Pearson's correlation analysis were used in the statistical analysis. Student-NewmanKeuls tests were used in the post hoc analysis. Interaction effects between the two groups and between the two scans revealing local neural activity as assessed by ALFF were observed in the bilateral dorsal stream visual cortex (DSVC), bilateral posterior cingulate cortex (PCC), bilateral anterior cingulate and medial prefrontal cortex (ACC and MPC), left temporo-parieto-occipital junction (TPOJ), left orbital and polar frontal cortex (OPFC), left paracentral lobular and mid cingulate cortex (PCL and MCC), right insular and frontal opercular cortex (IFOC), and left early visual cortex (EVC). Importantly, local functional activity in the left TPOJ and right PCC in the patient group was significantly lower than that in the HCs at baseline and was increased to relatively normal levels after treatment. The 24-week sound therapy tinnitus group demonstrated significantly higher ALFF in the left TPOJ and right PCC than in the tinnitus baseline group. Also, compared with the $\mathrm{HC}$ baseline group and the 24-week 
HC group, the 24-week sound therapy tinnitus group demonstrated slightly lower or higher ALFF in the left TPOJ and right PCC, and there were no differences between the 24-week sound therapy tinnitus and HC groups. Decreased THI scores and ALFF changes in the abovementioned brain regions were not correlated. Taken together, surface-based RS-fMRI can provide more subtle local functional activity to explain the mechanism of tinnitus treatment, and long-term sound therapy had a normalizing effect on tinnitus patients.

Keywords: tinnitus, sound therapy, surface-based analysis, ALFF, functional magnetic resonance imaging

\section{INTRODUCTION}

Tinnitus is a conscious awareness of a sound in the absence of any external acoustic stimulation, which is a major health issue in society worldwide (Bauer, 2018; Esmaili and Renton, 2018; Conlon et al., 2020). Noise trauma is the most common cause of subjective tinnitus (Grossan and Peterson, 2021), and it can trigger many related complications, such as insomnia, anxiety, depression; these complications seriously affect patients' quality of life (Bhatt et al., 2016; Sereda et al., 2018). An increasing number of studies have shown that tinnitus, as an abnormality of the central nervous system, can lead to significant alterations in brain structure and function, such as the emotional (amygdala, anterior insula), temporofrontal/stress-regulating regions (prefrontal cortex, inferior frontal gyrus); Functional connectivity revealed increased neural coupling between several auditory areas and non-auditory areas (amygdala, cerebellum, reticular formation, hippocampus, and caudate/putamen) (Hofmeier et al., 2018; Salvi et al., 2021). These alterations are closely related to the clinical characteristics of tinnitus patients and may even be the main cause of tinnitus (Han et al., 2020; Chen et al., 2021; Wei et al., 2021). However, due to the limitations of current research methods, it is still unclear which neural pathways or brain regions play a major role in tinnitus (Vanneste et al., 2019). This may also be the reason current treatment interventions cannot achieve satisfactory results for all tinnitus patients (Langguth et al., 2013). Therefore, effective and precise treatments for certain brain regions or nerve pathways are still urgently needed.

Many methods of treating tinnitus, such as repetitive transcranial magnetic stimulation (rTMS) (Poeppl et al., 2018), drug therapy (Zenner et al., 2017), tinnitus counseling and cognitive behavioral therapy (CBT) (Langguth et al., 2013), hearing aids (Yakunina et al., 2019), cochlear implants (Olze, 2015), and tinnitus retraining therapy, have been widely used clinically (Lee et al., 2019). According to the clinical practice guidelines for tinnitus, sound therapy is one of the recommended treatment methods (Tunkel et al., 2014). During this treatment, the generated sound will be set based on tinnitus features, including its pitch, loudness, and minimum masking level. This sound reduces the contrast between the tinnitus and the environment, diminishes sensitivity to tinnitus, and promotes habituation to the tinnitus sensation (Makar et al., 2017). Narrow-band noise sound therapy has been one of the commonly used methods for the treatment of tinnitus in recent years
(Henry et al., 2002). Narrow-band noise sound therapy has become the most widely used sound treatment method in our hospital and research center. In our study, narrow band sound was defined as a sound center frequency of $400 \mathrm{~Hz}$ or higher: the $1 / 3$ octave band is the narrowest and the $1 / 2$ octave band is the widest. Previous studies have demonstrated that sound therapy can alter brain function to achieve successful clinical treatment (Han et al., 2019a,b). However, previous studies were volumebased studies. The major problem in volume-based studies was that the accuracy of cortical positioning was insufficient (Coalson et al., 2018). As a result, to enhance the accuracy of cortical positioning, we adopted surface-based analysis in this study.

Different from traditional volume-based analysis, a novel 360-area surface-based cortical segmentation was applied for multimodal data using the Human Connectome Project (HCP) (Coalson et al., 2018). In this template, each cortical area has multiple characteristics, such as those representing connectivity, structure, function, auditory or visual maps (Coalson et al., 2018). Surface-based information mapping is a more sensitive measure of local information content and has better spatial selectivity and higher accuracy than other methods (Oosterhof et al., 2011). For example, surface-based analysis distinguishes between information about finger presses in the primary motor and somatosensory cortex, which can result in good spatial selectivity (Oosterhof et al., 2011). Based on the HCP template and surface-based analysis, we can extract and analyze various brain functional features, such as amplitude of lowfrequency fluctuation (ALFF), regional homogeneity (ReHo), and degree centrality. In the tinnitus research field, volumebased analysis has been used in some tinnitus studies on the microstructure of the brains of tinnitus patients (Tae et al., 2018; Besteher et al., 2019). Some studies have reported microstructural changes in the brains of tinnitus patients with voxel-based morphometry (VBM) (Husain et al., 2011). Our previous study also used VBM to evaluate tinnitus patients and found that the thalamus, as a deep gray matter (GM) nucleus, was significantly different in these patients after sound therapy (Wei et al., 2020). Some studies have investigated microstructural changes such as GM and white matter (WM) volume and thickness of tinnitus patients with surface-based morphometry (SBM) (Allan et al., 2016; Meyer et al., 2016). In another study, we began to explore microstructural changes at the surface-based cortical level in tinnitus patients before and after sound therapy (Wei et al., 2021). However, no one has studied the cortical changes such as alterations in 
cortical ALFF, at the surface-based functional level in tinnitus patients. Pertinently, we think it is very important to fully understand the relationship between the cortical function of brain regions and tinnitus.

Amplitude of low-frequency fluctuation is a common method used to analyze resting-state functional magnetic resonance imaging (RS-fMRI) data (Kublbock et al., 2014); this form of assessment uses voxel-based analysis and focuses on the power of blood oxygen level-dependent (BOLD) signals within the low-frequency range. The advantage of this analysis lies in the ability to reflect the intensity of local neural activity and analyze the relationship between brain regions (Zang et al., 2007). ALFF has been widely used in related studies of the diagnosis and treatment of tinnitus (Chen et al., 2014, 2015; Cai et al., 2020). Previous studies have demonstrated that tinnitus is characterized by abnormal RS-fMRI findings (Han et al., 2015; Lv et al., 2016a,b, 2018b). We used graphtheoretical methods and tract-based spatial statistics (TBSS) to investigate the associations between abnormal RS-fMRI findings and clinical variables ( $\mathrm{Lv}$ et al., 2018a; Han et al., 2019b; Chen et al., 2020). Other studies have also shown that tinnitus can cause significant changes in brain function and structure; such changes are closely related to clinical manifestations in patients (Ryu et al., 2016; Schmidt et al., 2017; Han et al., 2019a). However, these studies were limited to the volume-based level rather than the surface-based level in patients with tinnitus.

In this study, we utilized surface-based ALFF to reflect local neural activity in tinnitus patients who underwent 24 weeks of narrow-band noise sound therapy. We hypothesized that sound therapy could gradually restore local brain function to a relatively normal range, maybe involve some functional regions.

\section{MATERIALS AND METHODS}

\section{Standard Protocol Approval, Registration and Patient Consent}

This study was approved by the ethics committees of our research institution (Beijing Friendship Hospital, Capital Medical University, 2016-P2-012). Written informed consent was obtained from all study subjects.

\section{Subjects}

All patients and healthy volunteers were recruited from our institution. In this study, thirty-three patients with idiopathic tinnitus were enrolled. There were two patients with right laterality, five patients with left laterality, the rest had bilateral laterality. The tinnitus sound was described by patients as a persistent, low- or high-pitched sound in one or both ears. Sound more than $4 \mathrm{kHz}$ is considered high-frequency in clinical research. The inclusion criteria were as follows: 1865 years old, persistent idiopathic tinnitus (persistent for $\geq 6$ and $\leq 48$ months), right handedness, no significant hearing loss, and no history of associated brain diseases confirmed by conventional MRI. The exclusion criteria included neurological signs and/or a history of neurological disease; cardiovascular disease; pulsatile tinnitus, Meniere's disease, sudden deafness, or otosclerosis; claustrophobia; and inability to pitch-match their tinnitus. Twenty-six age-, sex-, education-, and handednessmatched healthy control (HC) subjects were enrolled as controls. The characteristics of the subjects are presented in Table 1.

\section{Sound Therapy and Clinical Evaluation}

A TinniTest $^{\circledR}$ (TTS, 1000A, China) comprehensive tinnitus diagnosis and treatment instrument was used for psychoacoustic testing. SpeechEasy eMasker ${ }^{\circledR}$ (Micro-DSP Technology Co., Ltd., China) was used to perform narrow-band sound therapy. We advised patients to use it in a quiet environment to achieve the best therapeutic effect. All of the enrolled tinnitus patients were examined for tinnitus loudness matching ( $\mathrm{L}=$ loudness of tinnitus), pitch matching ( $\mathrm{Tf}=$ tinnitus frequency), minimum masking level, and residual inhibition to characterize the patients' tinnitus and prepare them for treatment. Narrow-band sound therapy was administered to the participants in the tinnitus group for 24 weeks, three times a day for $20 \mathrm{~min}$ each time. For each tinnitus patient, the loudness of sound for treatment was set as L-5 $\mathrm{dB}$. The bandwidth was changed according to the center frequency, and the bandwidth was $1 / 3$ octave (for example, Tf $=3 \mathrm{kHz}$, low cut $=3 \mathrm{kHz} \times 2-6 / 1$, high cut $=3 \mathrm{kHz} \times 2^{6 / 1}$ ).

We also asked the patients to complete the Tinnitus Handicap Inventory (THI) to assess the severity of tinnitus before and after treatment. The primary outcome of this prospective study was the change in THI score after treatment. A reduction of at least 20 points in the THI score was considered effective treatment (Newman et al., 1998). No kind of sound was administered to the HC group during the study.

\section{Data Acquisition}

The functional imaging data were obtained from the tinnitus patients at baseline (without any treatment) and at the end of therapy (24th week). The HC group was also scanned at baseline and at the 24th week. Images were obtained using a 3.0T MRI system (Prisma, Siemens, Erlangen, Germany) with a 64-channel phase-array head coil. During the scanning process, we used suitable foam padding to minimize head motion, and we used earplugs to reduce scanner noise. All the participants were asked to stay awake, close their eyes, breathe evenly, and try to avoid any specific thoughts. We used a conventional brain axial $\mathrm{T} 1$ sequence before the scans to exclude individuals with any visible brain abnormalities. We obtained resting-state functional images of all participants using an echo-planar imaging (EPI) sequence. We required the subjects to remain still during the scan time and not to meditate. The scanning parameters were as follows: 33 axial slices with a slice thickness $=3.5 \mathrm{~mm}$ and interslice gap $=1 \mathrm{~mm}$, repetition time $(\mathrm{TR})=2000 \mathrm{~ms}$, echo time $(\mathrm{TE})=30 \mathrm{~ms}$, flip angle $(\mathrm{FA})=90^{\circ}$, bandwidth $=2368 \mathrm{~Hz} / \mathrm{Px}$, field of view $(\mathrm{FOV})=224 \mathrm{~mm} \times 224 \mathrm{~mm}$, and matrix $=64 \times 64$. The latter parameters resulted in an isotropic voxel size of $3.5 \mathrm{~mm} \times 3.5 \mathrm{~mm} \times 3.5 \mathrm{~mm}$. The total number of volumes was acquired in $8.06 \mathrm{~min}$. 
TABLE 1 | Demographic and clinical characteristics of participants.

\begin{tabular}{|c|c|c|c|c|c|}
\hline Characteristics & $\begin{array}{l}\text { Tinnitus patients } \\
\text { (baseline, } n=33 \text { ) }\end{array}$ & $\begin{array}{l}\text { Tinnitus patients } \\
\text { (24th week, } \\
n=33 \text { ) }\end{array}$ & $\begin{array}{l}\text { Healthy controls } \\
\text { (baseline, } n=26 \text { ) }\end{array}$ & $\begin{array}{l}\text { Healthy controls } \\
\text { (24th week, } \\
n=26)\end{array}$ & $P$ value \\
\hline Age (years,, $\bar{x} \pm s$ ) & $48.2 \pm 12.4$ & & $47.3 \pm 9.6$ & & $0.745^{a}$ \\
\hline Gender (male/female) & $23 / 10$ & & $15 / 11$ & & $>0.99^{b}$ \\
\hline Handedness & 33 right-handed & & 26 right-handed & & $>0.99^{a}$ \\
\hline Tinnitus duration (months) & $\geq 6$ and $\leq 48$ & & NA & & NA \\
\hline Tinnitus pitch & $250 \sim 8,000 \mathrm{~Hz}$ & & & & NA \\
\hline THI score & $52.5 \pm 44.3$ & $37.3 \pm 20.9$ & NA & NA & $0.011^{c}$ \\
\hline$\Delta \mathrm{THI}$ score & $15.3 \pm 32.8$ & NA & NA & NA & NA \\
\hline Laterality & $\begin{array}{c}2 \text { right, } 5 \text { left, } 26 \\
\text { bilateral }\end{array}$ & & & & \\
\hline
\end{tabular}

Data are presented as mean \pm standard deviation for all variables except gender.

THI: Tinnitus Handicap Inventory.

$\Delta T H I=$ THIpre-THIpost.

NA: not applicable.

a Two-sample t-tests.

${ }^{b}$ Chi-square test.

${ }^{c}$ Paired-samples t-tests.

\section{Pre-processing of Functional Data}

Data pre-processing was performed using DPABISurf ${ }^{1}$, a surfacebased RS-fMRI data analysis toolbox evolved from DPABISurf. DPABISurf uses the fMRIprep pipeline (Esteban et al., 2019) to pre-process the structural and functional MRI data and provides a set of statistical and viewing tools. The data preprocessing pipeline in the present study contained the following steps: (1) the initial five time points were discarded to allow for signal equilibration, (2) the data were converted into BIDS format (Gorgolewski and Poldrack, 2016), and then the fMRIPrep 1.5.0 docker was called. (3) Anatomical data pre-processing performed as follows: the T1-weighted (T1w) image was corrected for intensity non-uniformity (INU) with N4BiasFieldCorrection (Tustison et al., 2010) and was used as the T1w reference throughout the workflow. The T1w-reference was then skull-stripped with a Nipype implementation of the antsBrainExtraction workflow [from advanced normalization tools (ANTs)] (Avants et al., 2008) using OASIS30ANTs as the target template. Brain tissue segmentation of cerebrospinal fluid (CSF), WM, and GM was performed on brain-extracted T1w using fast (Zhang et al., 2001). Brain surfaces were reconstructed using recon-all (Dale et al., 1999). (4) Functional data preprocessing as follows: For each of the runs (resting-state) per subject, to achieve functional data pre-processing, the following steps were performed. First, a reference volume and its skullstripped version were generated using a custom fMRIPrep methodology. The BOLD reference was then coregistered to the T1w reference using bbregister (FreeSurfer), which implements boundary-based registration (Greve and Fischl, 2009). BOLD runs were slice-time corrected using 3dTshift (Cox and Hyde, 1997). The BOLD time series were resampled to surfaces on the fsaverage5 space. (5) Nuisance regression was conducted as follows: the Friston 24-parameter model (Friston et al., 1996) was used to regress out head motion confounds. Additionally,

\footnotetext{
${ }^{1}$ http://rfmri.org/DPABISurf
}

mean framework displacement (FD) was used to address the residual effects of motion in group analyses (Jenkinson et al., 2002). Other sources of spurious variance (WM and CSF signals) were also removed from the data through linear regression to reduce respiratory and cardiac effects. Additionally, linear trends were included as a regressor to account for drifts in the BOLD signal. (6) Finally, data were filtered and smoothed as follows: a bandpass temporal filter $(0.01-0.1 \mathrm{~Hz})$ and spatial smoothing [full-width at half-maximum (FWHM) of $6 \mathrm{~mm}$ ] were applied to the normalized functional images. After the above steps, we obtained cortical ALFF values.

\section{Statistical Analysis}

Demographic data were compared through two-sample $t$ tests and paired two-sample $t$ tests using SPSS 19.0 software (SPSS, Inc., Chicago, IL, United States). $P$ values $<0.05$ were considered statistically significant. Longitudinal changes in THI scores were also analyzed by using paired two-sample $t$ tests.

DPABISurf was used to pre-process the neuroimaging statistics. For cortical ALFF data, to determine the group $\times$ time interaction effect between the two groups and the two scans, the main effects of group (the tinnitus patient group and the HC group) and time (baseline and 24-week follow-up period), twoway mixed model analysis of variance (ANOVA) and post hoc analyses were performed. The brain regions showing significant time differences in the HC group were excluded (Wang et al., 2015). Cortical ALFF analyses were conducted using wholebrain analyses. A $P$ value of less than $0.05(P<0.025$ for each hemisphere) for cortical ALFF was considered statistically significant (Monte Carlo simulation corrected). We looked up tables of $P$ values based on simulations in which a $Z$ field was synthesized on the atlas surface. The tables were distributed in DPABISurf. The $P$ value for the cluster was determined by indexing the table based on the size of the cluster, the threshold used to form the cluster, and an estimate of the global FWHM. Clusters were extracted separately for both hemispheres. In 
post hoc analyses, Student-Newman-Keuls (SNK) tests were used for pairwise comparisons. Pearson's correlation analyses were further conducted to investigate the relationships between the change in cortical ALFF and 24 weeks of sound therapy of tinnitus patients [THI score $(\Delta \mathrm{THI}$ score $=$ THIpreTHIpost)]. $P<0.05$ was set as the threshold to determine significance. The cortical ALFF results were visualized with DPABISurf. Cortical ALFF was quantified from the T1w images

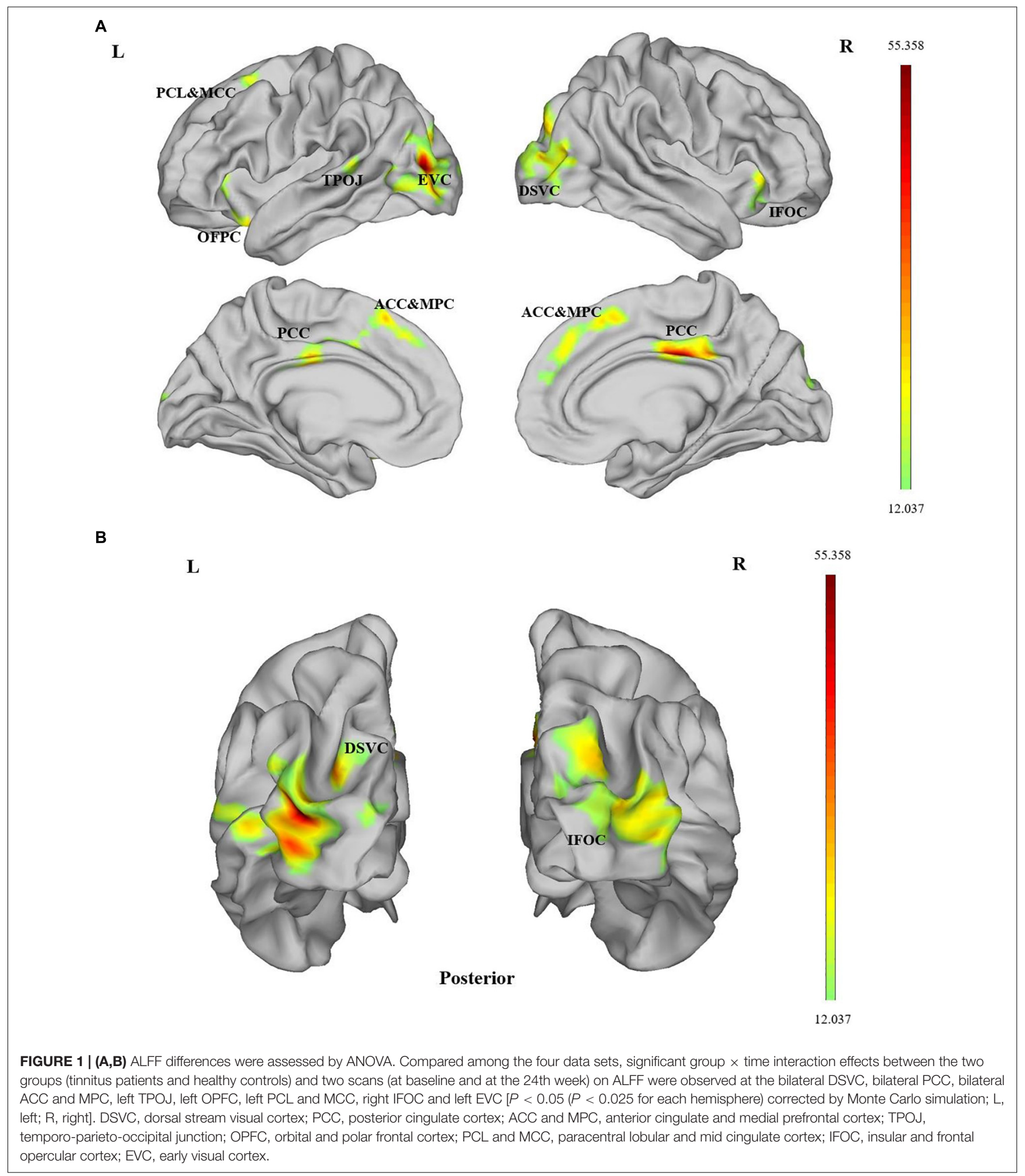


using DPABISurf_V1.5 software. Pearson's correlation analysis between the THI scores was performed using SPSS 19 software.

\section{RESULTS}

\section{Demographics and Behavioral Outcomes of Study Participants}

Please see Table 1, and we used DPABISurf to analyze cortical ALFF in the brains of patients in this group before and after sound therapy. After head movement correction, no subjects were excluded (FD $<0.3$ ). THI scores were acquired before and after sound therapy. Significant longitudinal decreases in THI scores were observed. The results are summarized in Table $\mathbf{1 .}$

\section{Statistical Analysis Results Group Differences in Amplitude of Low-Frequency Fluctuation}

As shown in Figure 1A and Table 2, there were differences in ALFF among the tinnitus patients before sound therapy (baseline), tinnitus patients after sound therapy (24 weeks), $\mathrm{HC}$ individuals at baseline and $\mathrm{HC}$ individuals after 24 weeks. The relevant brain regions included the bilateral dorsal stream visual cortex (DSVC), bilateral posterior cingulate cortex (PCC), bilateral anterior cingulate and medial prefrontal cortex (ACC and MPC), left temporo-parieto-occipital junction (TPOJ), left orbital and polar frontal cortex (OPFC), left paracentral lobular and mid cingulate cortex (PCL and MCC), right insular and frontal opercular cortex (IFOC), and left early visual cortex (EVC) $[P<0.05(P<0.025$ for each hemisphere $)$ corrected by Monte Carlo simulation] (Figures 1A,B).

\section{Post hoc Analyses}

Because other brain regions showing time differences in the $\mathrm{HC}$ group were excluded, only the left TPOJ and right PCC were retained for further analysis. Post hoc analysis in this study confirmed that patients with tinnitus all had decreased ALFF in related brain regions. ALFF was significantly decreased in the left TPOJ and right PCC of participants in the tinnitus baseline group compared to participants in the $\mathrm{HC}$ baseline group and HC 24-week group; also, there were no differences between the two HC groups (Figure 2). The 24-week sound therapy tinnitus group demonstrated significantly higher ALFF in the left TPOJ and right PCC than in the tinnitus baseline group (Figure 2).

Compared with the HC baseline group and the HC 24-week group, the 24-week sound therapy tinnitus group demonstrated slightly lower or higher ALFF in the left TPOJ and right PCC, and there were no differences between the 24-week sound therapy tinnitus group and either of the HC groups.

In post hoc analysis, there were no differences between the tinnitus baseline group and the 24-week sound therapy tinnitus group in the left DSVC, left OPFC, right ACC and MPC, right IFOC, and left EVC.

\section{Correlation}

Decreased THI scores and ALFF changes in the abovementioned brain regions were not correlated.

\section{DISCUSSION}

This is the first study to demonstrate cortical functional abnormality involving ALFF in patients with tinnitus. In this study, we analyzed the changes in cortical ALFF of tinnitus patients before and after 24 weeks of sound therapy. Cortical ALFF changes in the brain were found in patients from before and after longer-term sound therapy after multiple comparison corrections were conducted. Changes were mainly in the bilateral DSVC, bilateral PCC, bilateral ACC and MPC, left TPOJ, left OPFC, left PCL and MCC, right IFOC and EVC. Importantly,

TABLE 2 | Difference in cortical ALFF of the left and right hemisphere between the two groups (tinnitus patients, healthy controls) and two scans (at baseline, at the 24th week).

\begin{tabular}{|c|c|c|c|c|c|c|c|}
\hline \multirow[t]{2}{*}{ Brain regions } & & \multirow[t]{2}{*}{ HCP } & \multirow[t]{2}{*}{ Cluster Size $\left(\mathrm{mm}^{2}\right)$} & \multicolumn{3}{|c|}{ Coordinates MNI } & \multirow[t]{2}{*}{ Peak $F$ score } \\
\hline & & & & $x$ & $y$ & $z$ & \\
\hline \multirow[t]{2}{*}{ Dorsal stream visual cortex } & $\mathrm{L}$ & 16 & 336 & -24 & -81 & 23 & 34.64 \\
\hline & $\mathrm{R}$ & 13 & 2252 & 31 & -89 & 11 & 25.80 \\
\hline \multirow[t]{2}{*}{ Posterior cingulate cortex } & $\mathrm{L}$ & 14 & 241 & -6 & -17 & 32 & 32.15 \\
\hline & $\mathrm{R}$ & 32 & 668 & 6 & -19 & 32 & 48.79 \\
\hline \multirow[t]{2}{*}{ Anterior cingulate and medial prefrontal cortex } & $\mathrm{L}$ & 63 & 314 & -7 & 23 & 53 & 29.17 \\
\hline & $\mathrm{R}$ & 63 & 708 & 8 & 20 & 50 & 24.41 \\
\hline Temporo-parieto-occipital junction & $\mathrm{L}$ & 139 & 237 & -63 & -42 & 11 & 20.51 \\
\hline Orbital and polar frontal cortex & $\mathrm{L}$ & 94 & 385 & -26 & 14 & -20 & 25.88 \\
\hline Paracentral lobular and mid cingulate cortex & $\mathrm{L}$ & 41 & 175 & -11 & 6.1 & 37 & 18.51 \\
\hline Insular and frontal opercular cortex & $\mathrm{R}$ & 111 & 500 & 29 & 27 & 0 & 21.78 \\
\hline Early visual cortex & $\mathrm{L}$ & 6 & 2265 & -33 & -82 & 8 & 55.36 \\
\hline
\end{tabular}

Statistically differences in cortical ALFF were defined as $P<0.05$ ( $P<0.025$ for each hemisphere), Monte Carlo Simulation corrected after correcting for age, sex, education, and the head motion.

MNI, Montreal Neurological Institute; HCP, Human Connectome Project. 


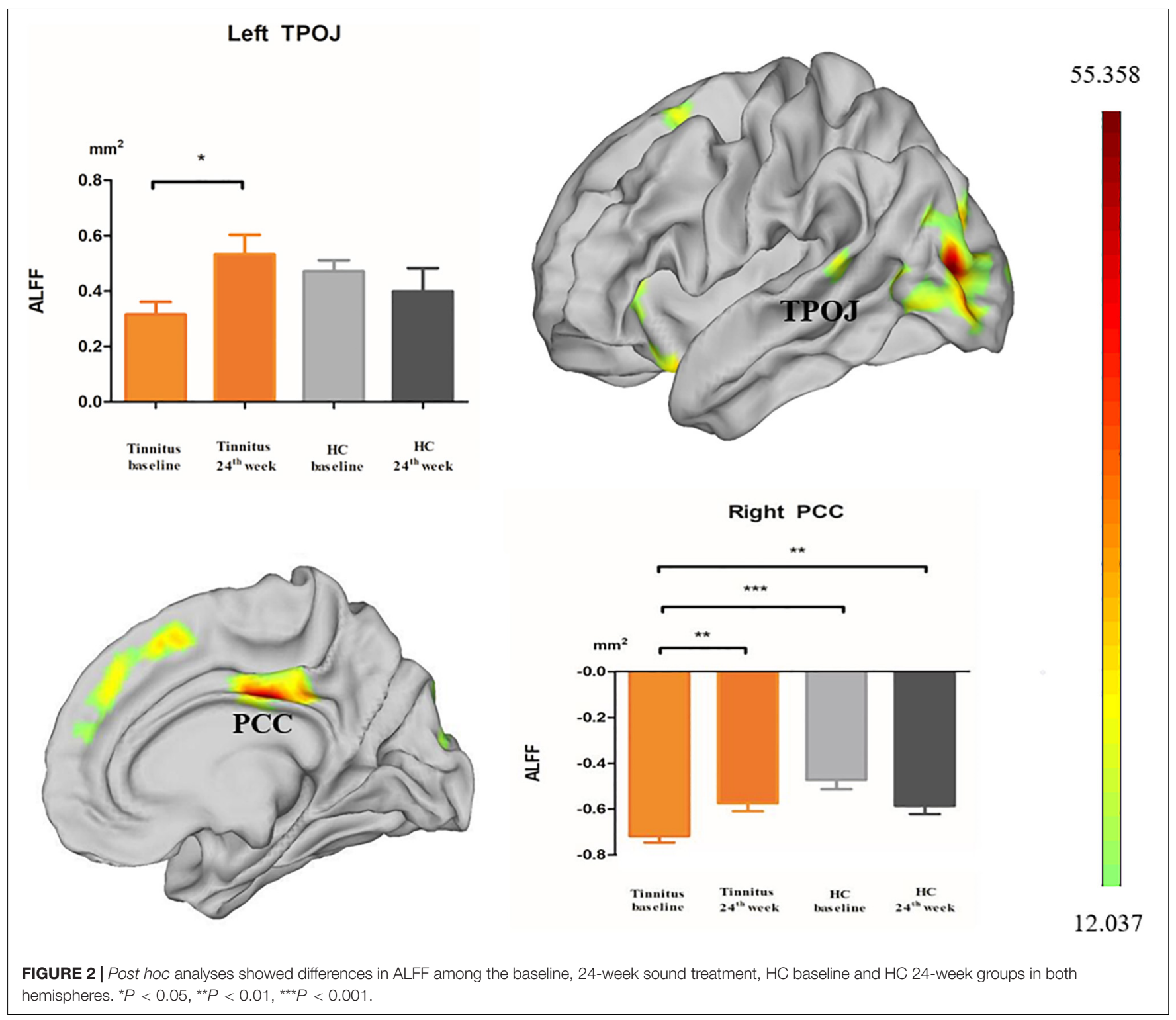

cortical ALFF changes in the left TPOJ and right PCC remained significant after post hoc analysis. These brain regions are mainly concentrated in the auditory cortex, visual cortex and PCC, which are closely related to the abnormal brain local neural activity associated with tinnitus. Our data confirmed our hypothesis that sound therapy could gradually restore brain function, especially local neural activity, to a relatively normal range.

We included patients whose tinnitus duration less than 48 months. Because of shorter duration may help with treatment effects (Schoisswohl et al., 2021). In our study, we observed that the 24-week sound therapy tinnitus group demonstrated significantly higher ALFF in the right PCC than in the tinnitus baseline group. As an important nucleus in the limbic system, the cingulate gyrus is widely connected with other areas of the central nervous system; moreover, it participates in various functions, such as regulating emotions, learning, and cognition (Fan et al., 2020). The PCC is an important structure of the default mode network (DMN) (Raichle and Snyder, 2007; Lan et al., 2021) and belongs to the frontal-parietal-limbic network, which has been regarded as a specific distress network in tinnitus (Husain, 2016). Abnormalities in the PCC are usually related to cognitive impairment, including memory function, attention, and problems in maintaining a balance between internal and external thinking (Leech and Sharp, 2014). The complete symptomology of tinnitus includes at least three factors: sensation, emotion, and cognition. Research on tinnitus has shown that abnormal changes in the cingulate cortex are involved in the process of tinnitus and play a key role in noise canceling, cognitive experiences and emotional experiences in tinnitus patients (Fan et al., 2020). "Dysfunctional noise canceling mechanism" has been conceptualized (De Ridder et al., 2012). A previous study found that some noise-canceling related effects in the subcallosal area (Rauschecker et al., 2010). The results of another study may designate the role of the rostral 
ACC as the core of the descending noise cancelation system (Song et al., 2015). These results all suggested the possibility of tinnitus perception modulation by neuromodulatory approaches to change the activity of above brain regions.

According to a previous report, the PCC undergoes significant changes in functional activity that ameliorate patient's pain after tinnitus treatment (Krick et al., 2017). In this study, the PCC was considered a hub brain region of the DMN, and ALFF became normal after sound therapy, reflecting that activation of the PCC was enhanced after treatment. This result was consistent with previous research on volume-based ALFF before and after treatment (Zhang A. et al., 2019). The results also showed that there were no differences between the 24-week sound therapy tinnitus group and HC groups in the PCC, showing that after sound therapy, cognition, attention, emotion and other related functions may be restored to a certain extent.

The HCP template identified the TPOJ as a strip of cortex bounded by the auditory, lateral temporal, inferior parietal and occipital cortices (Glasser et al., 2016). Although the TPOJ is not well known by many people, it contains the auditory cortex (lateral temporal) and visual cortex (occipital cortex), forming a bridge between advanced auditory and advanced visual areas (Glasser et al., 2016), which is closely related to tinnitus. Some previous volume-based RS-fMRI studies have shown that the functional connectivity between the visual network and the auditory network of tinnitus patients is negatively correlated. Tinnitus may also cause a decrease in spontaneous neural activity in the visual area (Burton et al., 2012; Maudoux et al., 2012). Through surface-based RS-fMRI, our research also inferred that the function of the auditory and visual cortices of patients with tinnitus maybe restored after long-term treatment.

In this study, we used surface-based ALFF analysis instead of volume-based ALFF analysis. Compared with surface-based methods, volume-based processing steps, especially smoothing and registration, significantly degrade cortical area localization; moreover, the spatial localization effect of the latter is only $35 \%$ of the best spatial positioning of the former (Coalson et al., 2018). As another neural activity indicator, cortical ReHo was demonstrated to be more specific to the intrinsic functional organization of the cortical mantle and had higher test-retest reliability (Zhang B. et al., 2019). However, in the tinnitus field, there is no research on the use of ALFF in the cortex, especially for comparisons before and after treatment. Therefore, our results proved the effectiveness of cortical functional imaging after tinnitus therapy to a certain extent and could more accurately reflect local functional changes at the cortical level. In addition, we used 24 weeks of sound therapy to evaluate patients after a relatively long period of time in this study. Compared with the shorter treatment period of 12 weeks used in previous studies (Wei et al., 2020; Lv et al., 2021), 24 weeks of treatment was more effective. With the extended treatment time, we obtained more detailed cortical information.

\section{Limitations}

First, there was no relationship between decreased THI scores and ALFF changes in tinnitus patients, and only left TPOJ and right PCC showed differences between the tinnitus baseline group and the 24-week sound therapy tinnitus group in the post hoc analysis; this outcome may have been due to the small sample size. In future studies, we need to further expand the sample size to verify the correlation between the differences observed. Second, few studies have reported treatment effects for tinnitus at the surface-based level, especially with ALFF. There are few related papers for our reference. Therefore, we need to combine other surface-based indicators, such as ReHo, degree centrality, and functional ALFF (fALFF), to study their significance more comprehensively. Third, the treatment time of 24 weeks should be gradually increased while considering the patient's tolerance. After all, achieving a longer period of clinical treatment is conducive to the recovery from tinnitus.

\section{CONCLUSION}

Longer-term sound therapy changed cortical ALFF in left TPOJ and right PCC. Surface-based RS-fMRI can provide more subtle local functional activity to explain the mechanism of tinnitus treatment, and these brain regions could serve as potential targets in the brain for neuroimaging evaluation of sound therapy in tinnitus patients. Notably, long-term sound therapy had a normalizing effect on tinnitus patients. Future follow-up studies in a larger cohort may elucidate further meaning of the changes in ALFF value after tinnitus.

\section{DATA AVAILABILITY STATEMENT}

The original contributions presented in the study are included in the article/supplementary material, further inquiries can be directed to the corresponding author/s.

\section{ETHICS STATEMENT}

The studies involving human participants were reviewed and approved by this research involved human participants. All authors have declared that this research was approved by the Institutional Review Board (IRB) of Beijing Friendship Hospital, Capital Medical University, Beijing, China. The patients/participants provided their written informed consent to participate in this study. Written informed consent was obtained from the individual(s) for the publication of any potentially identifiable images or data included in this article.

\section{AUTHOR CONTRIBUTIONS}

XW designed the experiments, performed the statistical analysis, and wrote the manuscript. QC conducted the statistical analysis. $\mathrm{PZ}$ contributed to the manuscript revision. HL, ZW, and CL collected the data. SG, ZY, and ZcW were guarantors of this work. $\mathrm{HL}$ and $\mathrm{ZcW}$ have full access to all the data in the study and take responsibility for the integrity of the data and the accuracy of the 
data analysis. All authors contributed to the article and approved the submitted version.

\section{FUNDING}

This work was supported by Grant Nos 62171297, 82171886, and 61931013 from the National Natural Science Foundation of China, Grant No. (2015) 160 from Beijing Scholars Program, Grant No. 7182044 from Beijing Natural Science Foundation, Grant No. 2020M680607 from China Postdoctoral Science Foundation, Grant No. 2021-zz-008 from Beijing Postdoctoral

\section{REFERENCES}

Allan, T. W., Besle, J., Langers, D. R., Davies, J., Hall, D. A., Palmer, A. R., et al. (2016). Neuroanatomical Alterations in Tinnitus Assessed with Magnetic Resonance Imaging. Front. Aging Neurosci. 8:221. doi: 10.3389/fnagi.2016. 00221

Avants, B. B., Epstein, C. L., Grossman, M., and Gee, J. C. (2008). Symmetric diffeomorphic image registration with cross-correlation: evaluating automated labeling of elderly and neurodegenerative brain. Med. Image Anal. 12, 26-41. doi: 10.1016/j.media.2007.06.004

Bauer, C. A. (2018). Tinnitus. N. Engl. J. Med. 378, 1224-1231. doi: 10.1056/ NEJMcp1506631

Besteher, B., Gaser, C., Ivansic, D., Guntinas-Lichius, O., Dobel, C., and Nenadic, I. (2019). Chronic tinnitus and the limbic system: Reappraising brain structural effects of distress and affective symptoms. Neuroimage Clin. 24:101976. doi: 10.1016/j.nicl.2019.101976

Bhatt, J. M., Lin, H. W., and Bhattacharyya, N. (2016). Prevalence, Severity, Exposures, and Treatment Patterns of Tinnitus in the United States. JAMA Otolaryngol. Head Neck Surg. 142, 959-965. doi: 10.1001/jamaoto.2016.1700

Burton, H., Wineland, A., Bhattacharya, M., Nicklaus, J., Garcia, K. S., and Piccirillo, J. F. (2012). Altered networks in bothersome tinnitus: a functional connectivity study. BMC Neurosci. 13:3. doi: 10.1186/1471-2202-13-3

Cai, Y., Xie, M., Su, Y., Tong, Z., Wu, X., Xu, W., et al. (2020). Aberrant Functional and Causal Connectivity in Acute Tinnitus With Sensorineural Hearing Loss. Front. Neurosci. 14:592. doi: 10.3389/fnins.2020.00592

Chen, Q., Lv, H., Wang, Z., Wei, X., Zhao, P., Yang, Z., et al. (2021). Outcomes at 6 months are related to brain structural and white matter microstructural reorganization in idiopathic tinnitus patients treated with sound therapy. Hum. Brain Mapp. 42, 753-765. doi: 10.1002/hbm.25260

Chen, Q., Wang, Z., Lv, H., Zhao, P., Yang, Z., Gong, S., et al. (2020). Reorganization of Brain White Matter in Persistent Idiopathic Tinnitus Patients Without Hearing Loss: Evidence From Baseline Data. Front. Neurosci. 14:591. doi: 10. 3389/fnins.2020.00591

Chen, Y. C., Li, X., Liu, L., Wang, J., Lu, C. Q., Yang, M., et al. (2015). Tinnitus and hyperacusis involve hyperactivity and enhanced connectivity in auditorylimbic-arousal-cerebellar network. Elife 4:e06576. doi: 10.7554/eLife.06576

Chen, Y. C., Zhang, J., Li, X. W., Xia, W., Feng, X., Gao, B., et al. (2014). Aberrant spontaneous brain activity in chronic tinnitus patients revealed by resting-state functional MRI. Neuroimage Clin. 6, 222-228. doi: 10.1016/j.nicl.2014.09.011

Coalson, T. S., Van Essen, D. C., and Glasser, M. F. (2018). The impact of traditional neuroimaging methods on the spatial localization of cortical areas. Proc. Natl. Acad. Sci. U S A 115, E6356-E6365. doi: 10.1073/pnas.1801582115

Conlon, B., Langguth, B., Hamilton, C., Hughes, S., Meade, E., Connor, C. O., et al. (2020). Bimodal neuromodulation combining sound and tongue stimulation reduces tinnitus symptoms in a large randomized clinical study. Sci. Transl. Med. 12:564. doi: 10.1126/scitranslmed.abb2830

Cox, R. W., and Hyde, J. S. (1997). Software tools for analysis and visualization of fMRI data. NMR Biomed. 10, 171-178. doi: 10.1002/(sici)1099-1492(199706/ 08) $10: 4 / 5<171:$ :aid-nbm $453<3.0$. co; $2-1$

Dale, A. M., Fischl, B., and Sereno, M. I. (1999). Cortical surface-based analysis. I. Segmentation and surface reconstruction. Neuroimage 9, 179-194. doi: 10.1006/ nimg.1998.0395
Research Foundation, and Grant No. yyqdkt2019-31 from Research Foundation of Beijing Friendship Hospital, Capital Medical University.

\section{ACKNOWLEDGMENTS}

We especially thank Beijing Friendship Hospital, Capital Medical University, for their support with our research. We also thank doctors from the Department of Otolaryngology Head and Neck Surgery, Beijing Friendship Hospital, Capital Medical University, for their assistance with the data collection and audiological tests.

De Ridder, D., Vanneste, S., Menovsky, T., and Langguth, B. (2012). Surgical brain modulation for tinnitus: the past, present and future. J. Neurosurg. Sci. 56, $323-340$.

Esmaili, A. A., and Renton, J. (2018). A review of tinnitus. Aust. J. Gen. Pract. 47, 205-208. doi: 10.31128/AJGP-12-17-4420

Esteban, O., Markiewicz, C. J., Blair, R. W., Moodie, C. A., Isik, A. I., Erramuzpe, A., et al. (2019). fMRIPrep: a robust preprocessing pipeline for functional MRI. Nat. Methods 16, 111-116. doi: 10.1038/s41592-0180235-4

Fan, X., Song, Y., and Ma, F. (2020). [The physiological function of cingulate cortex and its role in the mechanism of tinnitus]. Lin Chung Er Bi Yan Hou Tou Jing Wai Ke Za Zhi 34, 1141-1144. doi: 10.13201/j.issn.2096-7993.2020. 12.021

Friston, K. J., Williams, S., Howard, R., Frackowiak, R. S., and Turner, R. (1996). Movement-related effects in fMRI time-series. Magn. Reson. Med. 35, 346-355. doi: $10.1002 / \mathrm{mrm} .1910350312$

Glasser, M. F., Coalson, T. S., Robinson, E. C., Hacker, C. D., Harwell, J., Yacoub, E., et al. (2016). A multi-modal parcellation of human cerebral cortex. Nature 536, 171-178. doi: 10.1038/nature18933

Gorgolewski, K. J., and Poldrack, R. A. (2016). A Practical Guide for Improving Transparency and Reproducibility in Neuroimaging Research. PLoS Biol. 14:e1002506. doi: 10.1371/journal.pbio.1002506

Greve, D. N., and Fischl, B. (2009). Accurate and robust brain image alignment using boundary-based registration. Neuroimage 48, 63-72. doi: 10.1016/j. neuroimage.2009.06.060

Grossan, M., and Peterson, D. C. (2021). Tinnitus. Treasure Island,FL: StatPearls.

Han, L., Na, Z., Chunli, L., Yuchen, C., Pengfei, Z., Hao, W., et al. (2019a). Baseline Functional Connectivity Features of Neural Network Nodes Can Predict Improvement After Sound Therapy Through Adjusted Narrow Band Noise in Tinnitus Patients. Front. Neurosci. 13:614. doi: 10.3389/fnins.2019. 00614

Han, L., Yawen, L., Hao, W., Chunli, L., Pengfei, Z., Zhengyu, Z., et al. (2019b). Effects of sound therapy on resting-state functional brain networks in patients with tinnitus: A graph-theoretical-based study. J. Magn. Reson. Imaging 50, 1731-1741. doi: 10.1002/jmri.26796

Han, L., Pengfei, Z., Chunli, L., Zhaodi, W., Xindi, W., Qian, C., et al. (2020). The effects of sound therapy in tinnitus are characterized by altered limbic and auditory networks. Brain Commun. 2:fcaa131. doi: 10.1093/braincomms/ fcaa 131

Han, L., Zhaohui, L., Fei, Y., Pengfei, Z., Ting, L., Cheng, D., et al. (2015). Disrupted neural activity in unilateral vascular pulsatile tinnitus patients in the early stage of disease: evidence from resting-state fMRI. Prog. Neuropsychopharmacol. Biol. Psychiatry 59, 91-99. doi: 10.1016/j.pnpbp.2015.01.013

Henry, J. A., Schechter, M. A., Nagler, S. M., and Fausti, S. A. (2002). Comparison of tinnitus masking and tinnitus retraining therapy. J. Am. Acad. Audiol. 13, $559-581$.

Hofmeier, B., Wolpert, S., Aldamer, E. S., Walter, M., Thiericke, J., Braun, C., et al. (2018). Reduced sound-evoked and resting-state BOLD fMRI connectivity in tinnitus. Neuroimage Clin. 20, 637-649. doi: 10.1016/j.nicl.2018. 08.029

Husain, F. T. (2016). Neural networks of tinnitus in humans: Elucidating severity and habituation. Hear. Res. 334, 37-48. doi: 10.1016/j.heares.2015.09.010 
Husain, F. T., Medina, R. E., Davis, C. W., Szymko-Bennett, Y., Simonyan, K., Pajor, N. M., et al. (2011). Neuroanatomical changes due to hearing loss and chronic tinnitus: a combined VBM and DTI study. Brain Res. 1369, 74-88. doi: 10.1016/j.brainres.2010.10.095

Jenkinson, M., Bannister, P., Brady, M., and Smith, S. (2002). Improved optimization for the robust and accurate linear registration and motion correction of brain images. Neuroimage 17, 825-841. doi: 10.1016/s10538119(02)91132-8

Krick, C. M., Argstatter, H., Grapp, M., Plinkert, P. K., and Reith, W. (2017). Heidelberg Neuro-Music Therapy Enhances Task-Negative Activity in Tinnitus Patients. Front. Neurosci. 11:384. doi: 10.3389/fnins.2017. 00384

Kublbock, M., Woletz, M., Hoflich, A., Sladky, R., Kranz, G. S., Hoffmann, A., et al. (2014). Stability of low-frequency fluctuation amplitudes in prolonged resting-state fMRI. Neuroimage 103, 249-257. doi: 10.1016/j.neuroimage.2014. 09.038

Lan, L., Li, J., Chen, Y., Chen, W., Li, W., Zhao, F., et al. (2021). Alterations of brain activity and functional connectivity in transition from acute to chronic tinnitus. Hum. Brain Mapp. 42, 485-494. doi: 10.1002/hbm.25238

Langguth, B., Kreuzer, P. M., Kleinjung, T., and De Ridder, D. (2013). Tinnitus: causes and clinical management. Lancet Neurol. 12, 920-930. doi: 10.1016/ S1474-4422(13)70160-1

Lee, S. Y., Rhee, J., Shim, Y. J., Kim, Y., Koo, J. W., De Ridder, D., et al. (2019). Changes in the Resting-State Cortical Oscillatory Activity 6 Months After Modified Tinnitus Retraining Therapy. Front. Neurosci. 13:1123. doi: 10.3389/ fnins.2019.01123

Leech, R., and Sharp, D. J. (2014). The role of the posterior cingulate cortex in cognition and disease. Brain 137(Pt 1), 12-32. doi: 10.1093/brain/awt162

Lv, H., Chen, Q., Wei, X., Liu, C., Zhao, P., Wang, Z., et al. (2021). Sound therapy can modulate the functional connectivity of the auditory network. Prog. Neuropsychopharmacol. Biol. Psychiatry 2021:110323. doi: 10.1016/j.pnpbp. 2021.110323

Lv, H., Zhao, P., Liu, Z., Li, R., Zhang, L., Wang, P., et al. (2016a). Abnormal restingstate functional connectivity study in unilateral pulsatile tinnitus patients with single etiology: A seed-based functional connectivity study. Eur. J. Radiol. 85, 2023-2029. doi: 10.1016/j.ejrad.2016.09.011

Lv, H., Zhao, P., Liu, Z., Wang, G., Zeng, R., Yan, F., et al. (2016b). Frequency-Dependent Neural Activity in Patients with Unilateral Vascular Pulsatile Tinnitus. Neural. Plast. 2016:4918186. doi: 10.1155/2016/491 8186

Lv, H., Zhao, P., Liu, Z., Liu, X., Ding, H., Liu, L., et al. (2018b). Lateralization effects on functional connectivity of the auditory network in patients with unilateral pulsatile tinnitus as detected by functional MRI. Prog. Neuropsychopharmacol. Biol. Psychiatry 81, 228-235. doi: 10.1016/j.pnpbp.2017. 09.020

Lv, H., Wang, Z., Tong, E., Williams, L. M., Zaharchuk, G., Zeineh, M., et al. (2018a). Resting-State Functional MRI: Everything That Nonexperts Have Always Wanted to Know. AJNR Am. J. Neuroradiol. 39, 1390-1399. doi: 10. 3174/ajnr.A5527

Makar, S. K., Mukundan, G., and Gore, G. (2017). Treatment of Tinnitus: A Scoping Review. Int. Tinnitus. J. 21, 144-156. doi: 10.5935/0946-5448.201 70027

Maudoux, A., Lefebvre, P., Cabay, J. E., Demertzi, A., Vanhaudenhuyse, A., Laureys, S., et al. (2012). Connectivity graph analysis of the auditory resting state network in tinnitus. Brain Res. 1485, 10-21. doi: 10.1016/j.brainres.2012. 05.006

Meyer, M., Neff, P., Liem, F., Kleinjung, T., Weidt, S., Langguth, B., et al. (2016). Differential tinnitus-related neuroplastic alterations of cortical thickness and surface area. Hear. Res. 342, 1-12. doi: 10.1016/j.heares.2016.08.016

Newman, C. W., Sandridge, S. A., and Jacobson, G. P. (1998). Psychometric adequacy of the Tinnitus Handicap Inventory (THI) for evaluating treatment outcome. J. Am. Acad. Audiol. 9, 153-160.

Olze, H. (2015). Cochlear implants and tinnitus. HNO 63, 291-297. doi: 10.1007/ s00106-014-2975-5

Oosterhof, N. N., Wiestler, T., Downing, P. E., and Diedrichsen, J. (2011). A comparison of volume-based and surface-based multi-voxel pattern analysis. Neuroimage 56, 593-600. doi: 10.1016/j.neuroimage.2010. 04.270
Poeppl, T. B., Langguth, B., Lehner, A., Frodl, T., Rupprecht, R., Kreuzer, P. M., et al. (2018). Brain stimulation-induced neuroplasticity underlying therapeutic response in phantom sounds. Hum. Brain Mapp. 39, 554-562. doi: 10.1002/ hbm. 23864

Raichle, M. E., and Snyder, A. Z. (2007). A default mode of brain function: a brief history of an evolving idea. Neuroimage 37, 1083-1090. doi: 10.1016/j. neuroimage.2007.02.041

Rauschecker, J. P., Leaver, A. M., and Muhlau, M. (2010). Tuning out the noise: limbic-auditory interactions in tinnitus. Neuron 66, 819-826. doi: 10.1016/j. neuron.2010.04.032

Ryu, C. W., Park, M. S., Byun, J. Y., Jahng, G. H., and Park, S. (2016). White matter integrity associated with clinical symptoms in tinnitus patients: A tract-based spatial statistics study. Eur. Radiol. 26, 2223-2232. doi: 10.1007/s00330-0154034-3

Salvi, R., Auerbach, B. D., Lau, C., Chen, Y. C., Manohar, S., Liu, X., et al. (2021). Functional Neuroanatomy of Salicylate- and Noise-Induced Tinnitus and Hyperacusis. Curr. Top. Behav. Neurosci. 51, 133-160. doi: 10.1007/7854_ 2020_156

Schmidt, S. A., Carpenter-Thompson, J., and Husain, F. T. (2017). Connectivity of precuneus to the default mode and dorsal attention networks: A possible invariant marker of long-term tinnitus. Neuroimage Clin. 16, 196-204. doi: 10.1016/j.nicl.2017.07.015

Schoisswohl, S., Schecklmann, M., Langguth, B., Schlee, W., and Neff, P. (2021). Neurophysiological correlates of residual inhibition in tinnitus: Hints for traitlike EEG power spectra. Clin. Neurophysiol. 132, 1694-1707. doi: 10.1016/j. clinph.2021.03.038

Sereda, M., Xia, J., El Refaie, A., Hall, D. A., and Hoare, D. J. (2018). Sound therapy (using amplification devices and/or sound generators) for tinnitus. Cochrane Database Syst. Rev. 12:CD013094. doi: 10.1002/14651858.CD013094. pub2

Song, J. J., Vanneste, S., and De Ridder, D. (2015). Dysfunctional noise cancelling of the rostral anterior cingulate cortex in tinnitus patients. PLoS One 10:e123538. doi: 10.1371/journal.pone.012 3538

Tae, W. S., Yakunina, N., Lee, W. H., Ryu, Y. J., Ham, H. K., Pyun, S. B., et al. (2018). Changes in the regional shape and volume of subcortical nuclei in patients with tinnitus comorbid with mild hearing loss. Neuroradiology 60, 1203-1211. doi: 10.1007/s00234-018-2093-2

Tunkel, D. E., Bauer, C. A., Sun, G. H., Rosenfeld, R. M., Chandrasekhar, S. S., Cunningham, E. R. Jr., et al. (2014). Clinical practice guideline: tinnitus. Otolaryngol. Head Neck Surg. 2(Suppl.), S1-S40. doi: 10.1177/ 0194599814545325

Tustison, N. J., Avants, B. B., Cook, P. A., Zheng, Y., Egan, A., Yushkevich, P. A., et al. (2010). N4ITK: improved N3 bias correction. IEEE Trans. Med. Imaging 29, 1310-1320. doi: 10.1109/TMI.2010.2046908

Vanneste, S., Alsalman, O., and De Ridder, D. (2019). Top-down and Bottomup Regulated Auditory Phantom Perception. J. Neurosci. 39, 364-378. doi: 10.1523/JNEUROSCI.0966-18.2018

Wang, L., Xia, M., Li, K., Zeng, Y., Su, Y., Dai, W., et al. (2015). The effects of antidepressant treatment on resting-state functional brain networks in patients with major depressive disorder. Hum. Brain Mapp. 36, 768-778. doi: 10.1002/ hbm. 22663

Wei, X., Lv, H., Chen, Q., Wang, Z., Liu, C., Zhao, P., et al. (2021). Cortical Thickness Alterations in Patients With Tinnitus Before and After Sound Therapy: A Surface-Based Morphometry Study. Front. Neurosci. 15:633364. doi: $10.3389 /$ fnins.2021.633364

Wei, X., Lv, H., Wang, Z., Liu, C., Ren, P., Zhang, P., et al. (2020). Neuroanatomical Alterations in Patients With Tinnitus Before and After Sound Therapy: A Voxel-Based Morphometry Study. Front. Neurosci. 14:911. doi: 10.3389/fnins. 2020.00911

Yakunina, N., Lee, W. H., Ryu, Y. J., and Nam, E. C. (2019). Tinnitus Suppression Effect of Hearing Aids in Patients With High-frequency Hearing Loss: A Randomized Double-blind Controlled Trial. Otol. Neurotol. 40, 865-871. doi: 10.1097/MAO.0000000000002315

Zang, Y. F., He, Y., Zhu, C. Z., Cao, Q. J., Sui, M. Q., Liang, M., et al. (2007). Altered baseline brain activity in children with ADHD revealed by restingstate functional MRI. Brain Dev. 29, 83-91. doi: 10.1016/j.braindev.2006. 07.002 
Zenner, H. P., Delb, W., Kroner-Herwig, B., Jager, B., Peroz, I., Hesse, G., et al. (2017). A multidisciplinary systematic review of the treatment for chronic idiopathic tinnitus. Eur. Arch. Otorhinolaryngol. 274, 2079-2091. doi: 10.1007/ s00405-016-4401-y

Zhang, A., Li, G., Yang, C., Liu, P., Wang, Y., Kang, L., et al. (2019). Alterations of amplitude of low-frequency fluctuation in treatment-resistant versus non-treatment-resistant depression patients. Neuropsychiatr. Dis. Treat 15, 2119-2128. doi: 10.2147/NDT.S1 99456

Zhang, B., Wang, F., Dong, H. M., Jiang, X. W., Wei, S. N., Chang, M., et al. (2019). Surface-based regional homogeneity in bipolar disorder: A restingstate fMRI study. Psychiatry Res. 278, 199-204. doi: 10.1016/j.psychres.2019. 05.045

Zhang, Y., Brady, M., and Smith, S. (2001). Segmentation of brain MR images through a hidden Markov random field model and the expectationmaximization algorithm. IEEE Trans. Med. Imaging 20, 45-57. doi: 10.1109/ 42.906424
Conflict of Interest: The authors declare that the research was conducted in the absence of any commercial or financial relationships that could be construed as a potential conflict of interest.

Publisher's Note: All claims expressed in this article are solely those of the authors and do not necessarily represent those of their affiliated organizations, or those of the publisher, the editors and the reviewers. Any product that may be evaluated in this article, or claim that may be made by its manufacturer, is not guaranteed or endorsed by the publisher.

Copyright (c) 2021 Wei, Lv, Chen, Wang, Zhao, Liu, Gong, Yang and Wang. This is an open-access article distributed under the terms of the Creative Commons Attribution License (CC BY). The use, distribution or reproduction in other forums is permitted, provided the original author(s) and the copyright owner(s) are credited and that the original publication in this journal is cited, in accordance with accepted academic practice. No use, distribution or reproduction is permitted which does not comply with these terms. 